\title{
Development and study of mechanical behaviour reinforcing composites by waste BTP
}

\section{Elaboration et étude du comportement mécanique de nouveaux composites renforcés par des déchets BTP}

\author{
M.El kanzaoui ${ }^{1}$, A.Hajjaji ${ }^{2}$, A.Guenbour ${ }^{1}$, R.Boussen ${ }^{1}$ \\ ${ }^{1}$ Université Mohamed V Rabat, Faculté des Sciences, Rabat, Maroc \\ ${ }^{2}$ Université Chouaib Doukkali , École Nationale des Sciences Appliquées, El Jadida, Maroc
}

\begin{abstract}
Composite materials are used in many industrial applications for their excellent mechanical and electric properties and their low density compared to metal structures. Most countries are extremely rich waste materials such as white ceramic breakages which represents a potential to be developed. Ceramic breakages have exceptional properties and could be effectively exploited in the manufacture of composite materials for a wide variety of applications. The composite materials reinforced by construction waste materials, such as ceramic breaks which offer significant benefits and gains in strength and stiffness properties (Young's modulus E : a material whose modulus Young is very high is said rigid). This article covers the benefits of breakages as ceramic filler used for reinforcement in composites, as well as improve the mechanical response of these structural elements (test compression).
\end{abstract}

Key words: composite materials, ceramics, construction materials, mechanical properties, compression test, Young's modulus

Résumé. Les matériaux composites sont utilisés dans de nombreuses applications industrielles pour leurs excellentes propriétés mécaniques et pour leur masse volumique faible par rapport à celles des structures métalliques. La majorité des pays sont extrêmement riches en déchets issue des différents procédés de fabrication industrielle telles que les casses de céramique qui représentent un potentiel à valoriser. Les casses de céramique ont des propriétés exceptionnelles et pourraient être exploitées efficacement dans la fabrication des matériaux composites pour une grande diversité d'applications. Les matériaux composites à renfort par des déchets matériaux BTP, comme les casses céramique qui offrent des avantages notables et des gains en termes de propriétés de résistance mécanique et de rigidité (Module d'Young E : un matériau dont le module d'Young est très élevé est dit rigide).Le présent article couvre les avantages des casses céramique comme charge utilisée pour le renforcement dans les composites, ainsi que d'améliorer la réponse mécanique de ces éléments structurels (Essai de compression).

Mots clés : matériaux composites, céramique, matériaux BTP, propriété mécanique, essai de compression, module d'Young 


\section{Introduction}

$\mathrm{Au}$ cours de ces dernières années, les industries des matériaux composites ont bénéficié d'une croissance rapide et régulière, soutenue en particulier par la diversité des applications.

Les matériaux composites sont des matériaux solides hétérogènes et anisotropes, constitués de l'assemblage d'au moins deux matériaux non miscibles et de natures différentes, se complétant et permettant d'aboutir à un matériau dont l'association confère à l'ensemble des propriétés qu'aucun des composants pris séparément ne possède [1]. Ils sont constitués d'une ou plusieurs phases discontinues reparties dans une phase continue. La phase continue est appelée matrice et la phase discontinue, qui présente usuellement des propriétés mécaniques (rigidités et résistances) supérieures à celle de la matrice, est nommée renfort.

Les matériaux composites offrent d'excellents rapports masse/rigidité/résistance en comparaison avec les matériaux métalliques. Ils répondent parfaitement aux exigences mécaniques, thermiques, acoustiques, transparence, résistance aux feux et fatigue [2-3].

Les matériaux composites sont développés et choisis pour leurs caractéristiques mécaniques, thermiques, ou chimiques. Les propriétés obtenues sont généralement meilleures que celles de la matrice seule. Bien évidemment, il est impossible de réunir l'ensemble de ces propriétés dans un même composite mais, par rapport aux caractéristiques recherchées, on peut trouver des solutions optimales.

Les composites ne cessent d'évoluer vers des produits qui sont, soit les moins coûteux possibles, soit les plus performants, ou bien les deux à la fois. De plus, dans un souci de protection de l'environnement et de santé publique, les composites tendent à intégrer un caractère écologique.

De nos jours, l'utilisation des déchets d'industrie matériaux dans les matériaux composites devient de plus en plus fréquente, et ce, du fait que ces ressources sont moins coûteuses et présentent une valeur ajoutée. De plus, les tendances industrielles se penchent vers des produits propres « inertes » et écologiques. D'où l'intérêt d'intégrer des produits à potentiel polluant pratiquement nul, non réactif, non évolutif, non ou peu soluble.

La valorisation des résidus de l'industrie minérale sont devenus extrêmement importants dans l'élaboration des matériaux innovants, écologiques, plus performants, et moins coûteux. L'essentiel de ces déchets est produit sur les chantiers du BTP et bien précisément les résidus de l'industrie céramique « la casse Rouge ».

La casse de céramique rouge représente un déchet solide minéral, non susceptible d'évolution physicochimique ou biologique. Il ne se décompose pas, ne brûle pas, n'est pas biodégradable.

Les céramiques qui sont en général durs et fragiles à la rupture tout en se caractérisant parfois par des résistances mécaniques très élevée, c'est pour cela qu'ils ont été utilisés en tant que matériaux de constructions depuis l'antiquité.
L'objectif principal de notre travail est la mise en œuvre des composites à matrice renforcée par des casses céramiques « résidu minéral », la caractérisation physico-chimique, la caractérisation morphologique et en fin le comportement mécanique des matériaux ainsi obtenus.

\section{Matériels et méthodes}

\subsection{Matière première}

Résine polyester insaturé (UP) thermodurcissable. Déchet industriel traité :

La casse céramique rouge (région de Casablanca)

\subsubsection{Préparation de la casse rouge}

L'échantillon de la casse rouge a subit les opérations unitaires suivantes

-concassage des fragments

-broyage des morceaux dans un broyeur

-tamisage de la poudre

\subsection{Elaboration du nouveau matériau composite CR}

Le matériau composite a été élaboré avec différentes teneurs en casse rouge brute $15 \%, 30 \%$ et $50 \%$.

Finement broyée, la casse était ensuite mélangée avec une matrice thermodurcissable (résine).

Le mélange obtenu est versé dans un moule cylindrique puis mis à durcir dans une étuve à $60^{\circ} \mathrm{C}$.

Après extraction du matériau on procède à la découpe de plusieurs échantillons avec des épaisseurs variables.

\section{3. Caractérisation des matériaux}

Une définition très générale d'une céramique pourrait être la suivante: une céramique est un matériau inorganique polycristallin, présentant une structure complexe de grains et de joints de grains [4].

Le terme générique de céramique recouvre des domaines aussi divers et variés que celui des céramiques traditionnelles (tuiles, briques, carreaux, ...) qui nous intéresse particulièrement, ou celui des céramiques dites techniques à applications thermomécaniques ou électroniques [5].

Les matériaux céramiques présentent une caractéristique essentielle par rapport à d'autres matériaux : les atomes ou constituants de leur réseau cristallin sont en général très solidement liés entre eux par des liaisons fortes (ioniques et covalentes) [6].

Les céramiques jouent actuellement un rôle de plus en plus important mettant à profit leurs propriétés électriques, électromécaniques, isolantes et optiques $[7,8]$.

Notre présent travail s'intéresse particulièrement à des rejets céramiques ou bien les casses céramiques précisément les casses rouges $\mathrm{CR}$. 
Les céramiques sont souvent des assemblages complexes de cristaux et de phase vitreuse. Seules certaines céramiques techniques, préparées à partir de produits de haute pureté, comportent une phase unique cristallisée. La nature et les proportions des constituants dépendent en premier lieu de la composition chimique du matériau de départ, mais sont aussi influencées par les traitements de cuisson et de refroidissement appliqués. Une céramique se caractérise à la fois par ses constituants et par sa microstructure, c'est-à-dire l'ensemble des paramètres décrivant l'organisation des différentes phases (taille et morphologie des cristaux et des phases inter granulaires, inclusions, porosités ouvertes ou fermées, etc.). [9]

La caractérisation des casses céramiques a été réalisée par spectrométrie IR, Microscopie et par diffraction des rayons $\mathrm{X}$.

\subsection{Microscopie optique à haute résolution}

L'observation au Microscope nous a permis de visualiser les surfaces, et de mettre en évidence les détails d'une grande finesse. L'observation a été réalisée avec microscope: digital microscope Leica DVM5000 HD.

\subsection{Spectroscopie Infrarouge (ATR)}

Les spectres infrarouge à transformée de Fourier (IRTF) ont été effectués à l'aide d'un appareil de type VERTEX 70 dans la gamme des longueurs d'onde de 400 cm-1 à 4000 cm-1; la résolution spectrale est de 4,0 cm-1.

\subsection{Diffractométrie des rayons $X$ (DRX)}

Les phases cristallines ont été identifiées par diffraction des rayons $\mathrm{X}$ sur des poudres avec un diffractomètre Siemens d5000 X-Ray à monochromateur arrière en graphite, fonctionnant sous une tension de 40 $\mathrm{kV}$ et une intensité de $50 \mathrm{~mA}$ avec la radiation $\mathrm{CuK \alpha}$ comme source de rayonnement et piloté par un ordinateur muni du logiciel Diffracplus D version 2.2.

\subsection{Module d'Young Essai de compression}

L'essai mécanique est utilisé pour étudier l'effet positif ou négatif de la charge « casse céramique » sur les propriétés du matériau composite CR.

Les matériaux composites élaborés, avec différentes teneurs en casse rouge $15 \%, 30 \%$ et $50 \%$, ont été découpés en des échantillons d'épaisseurs 6,10 et $15 \mathrm{~mm}$ comme décrit dans le tableau 1.
Tableau 1. Gamme d'échantillons matériau composite CR pour essai mécanique compression.

\begin{tabular}{|c|c|c|c|}
\hline \multirow{2}{*}{$\begin{array}{c}\text { Echantillon } \\
\text { Dimensions }\end{array}$} & CR $15 \%$ & CR30\% & CR $50 \%$ \\
\hline & & & \\
& D :20mm & D:20mm & D: $20 \mathrm{~mm}$ \\
& $\mathrm{E}: 6 \mathrm{~mm}$ & $\mathrm{E}: 6 \mathrm{~mm}$ & $\mathrm{E}: 6 \mathrm{~mm}$ \\
Diamètre & & & \\
\cline { 2 - 4 } (D) & $\mathrm{D}: 20 \mathrm{~mm}$ & $\mathrm{D}: 20 \mathrm{~mm}$ & $\mathrm{D}: 20 \mathrm{~mm}$ \\
(E) & $\mathrm{E}: 10 \mathrm{~mm}$ & $\mathrm{E}: 10 \mathrm{~mm}$ & $\mathrm{E}: 10 \mathrm{~mm}$ \\
& & & \\
\cline { 2 - 4 } & $\mathrm{D}: 20 \mathrm{~mm}$ & $\mathrm{D}: 20 \mathrm{~mm}$ & $\mathrm{D}: 20 \mathrm{~mm}$ \\
& $\mathrm{E}: 15 \mathrm{~mm}$ & $\mathrm{E}: 15 \mathrm{~mm}$ & $\mathrm{E}: 15 \mathrm{~mm}$ \\
& & & \\
& & & \\
\hline
\end{tabular}

Pour évaluer le module d'Young, trois échantillons étaient testés et la valeur moyenne déclarée. L'expérience a été réalisée sous charge quasi-statique de compression pour tous les échantillons en utilisant une machine d'essai mécanique normalisée, pilotée par un ordinateur muni du logiciel LM Acq2V version 1.05.

Les propriétés de compression, tel que, le module d' Young est obtenu à partir des courbes de compression avec régression linéaire en exploitant le logiciel Statgraphics.

Ces essais nous ont permis de déterminer le module d'Young E donnée par la formule suivante :

$\mathrm{E}=\mathrm{Fe} /$ So $\mathrm{x} \mathrm{Lo} / \Delta \mathrm{L}$

$\mathrm{E}=$ la pente de la droite*facteur de forme

Fe : force appliquée

So : section initiale

Lo : épaisseur initial

$\Delta \mathrm{L}$ : allongement

\section{Résultats et Discussions}

\subsection{Analyse de la casse rouge}

\subsubsection{Microscopie optique à haute résolution}

La microscopie permet d'observer la texture de la casse rouge et de caractériser les assemblages minéralogiques. L'image obtenue de l'échantillon avec l'agrandissement $200 \mu$, est représentée sur la figure 1. 


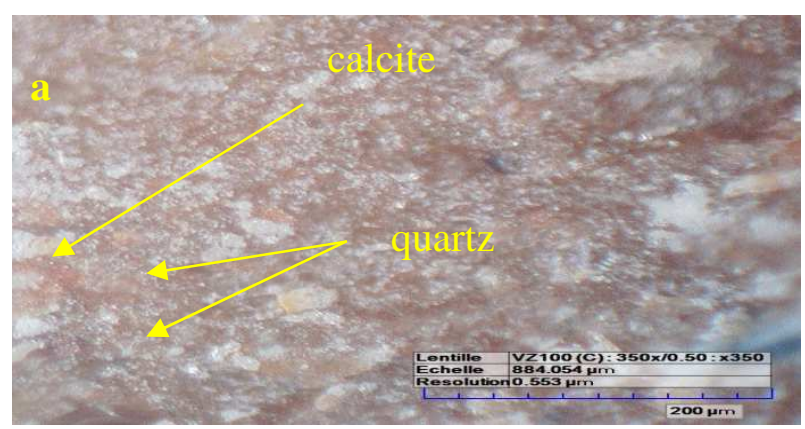

Fig. 1 Observations au microscope optique à haute résolution de la casse rouge.

Les particules argileuses se présentent sous forme d'amas d'agrégats fins et de plaquettes en forme des bâtonnets aux contours irréguliers (fig.1).

Il s'agit d'une morphologie rencontrée aussi bien pour des Kaolinites mal cristallisées que pour des Illites comme l'a observé Konan [10]. L'image de la figure 1 est en accord avec ce que nous avons obtenu en DRX, confirmant ainsi la présence de carbonates et de Quartz dans l'échantillon. Les carbonates (Calcite) se présentent sous forme des agrégats bien visibles et le Quartz se présente sous forme des grains de petite taille [11,12].

\subsubsection{Spectroscopie Infrarouge (ATR)}

Cette étude a permis de caractériser la casse rouge.

Tableau 2. Principales bandes IR caractéristiques de la casse rouge.

\begin{tabular}{|c|c|}
\hline Les bandes (cm-1) & Interprétations \\
\hline 1480 & (1400 et 1500$) \mathrm{cm}^{-1} \mathrm{CH} 3$. \\
\hline & $\begin{array}{l}\text { (900-1100)cm } \mathrm{cm}^{-1} \\
\text { d'allongement de Si-O (Kaolinite) }^{-}\end{array}$ \\
\hline 1000 & \\
\hline 795 & Liaisons $\mathrm{Si}-\mathrm{O}-\mathrm{Al}$ \\
\hline 400 & vibrations de déformation des $\mathrm{OH}$ \\
\hline
\end{tabular}

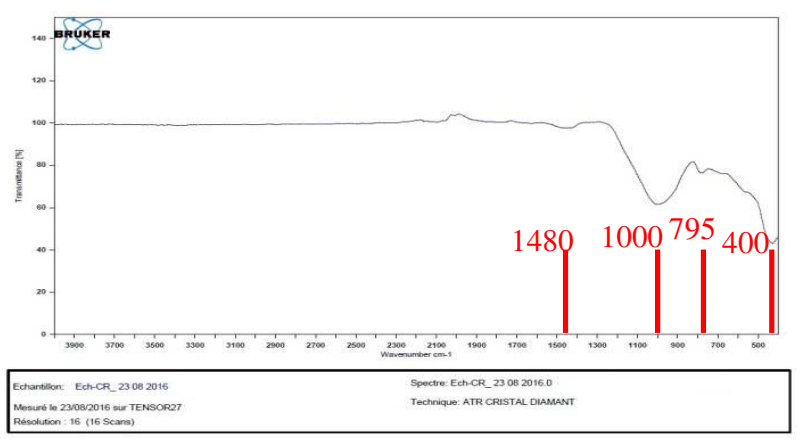

Fig. 2. Spectre infrarouge casse rouge CR.
Les spectres obtenus sont illustrés par la figure 2. On constate: La bande située entre 1400 et $1500 \mathrm{~cm}-1$ est attribuée aux vibrations de déformation des groupes $\mathrm{CH} 3$ situés à $1480 \mathrm{~cm}-1$. La liaison Si-O est caractérisée par : La bande intense située entre $900-1100 \mathrm{~cm}-1$ et centrée vers $1000 \mathrm{~cm}-1$ correspond aux vibrations de valence de la liaison $\mathrm{Si}-\mathrm{O}[13]$.

La bande entre 750 et $800 \mathrm{~cm}-1$, provenant de la liaison $\mathrm{Si}-\mathrm{O}-\mathrm{Al}$, fait également place à une bande autour de 795 cm-1. La bande d'absorption située à $1000 \mathrm{~cm}-1$ est en accord avec la DRX indiquant la présence de la Kaolinite dans notre matériau CR. La bande observée à $795 \mathrm{~cm}-1$ est attribuable aux vibrations d'élongation des liaisons $\mathrm{Si}-\mathrm{O}-\mathrm{Al}$ et aux hydroxyles perpendiculairement à la surface (OH translationnel) [14]. La bande à $400 \mathrm{~cm}-1$ est caractéristique des vibrations de déformation des hydroxyles dans les minéraux argileux trioctaédriques en général. Néanmoins, la bande d'absorption à 795 cm-1 peuvent correspondre au Quartz [15].

. Ces résultats sont en accord avec ceux de DRX. Ils confirment la présence du Quartz, de la Kaolinite et de l'Illite dans la casse rouge étudié. Le tableau 1, regroupe les bandes de vibrations et de déformations issues de la figure1.

\subsubsection{Diffractométrie des rayons $X(D R X)$}

Les diffractogrammes ont été obtenus à partir des échantillons désorientés (placés directement sous forme de poudre dans un porte-échantillon classique).

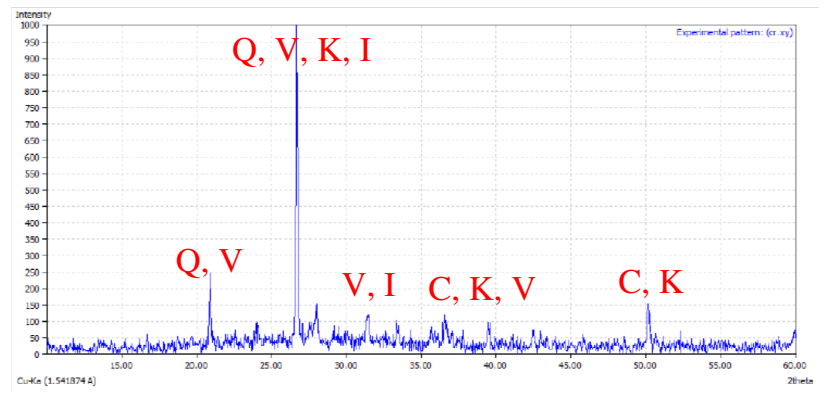

Fig. 3. Diffractogramme $X$ casse rouge $C R$, avec $K=$ Kaolinite, $\mathrm{I}=$ Illite, $\mathrm{Q}=$ Quartz, $\mathrm{C}=$ Calcite, $\mathrm{V}=$ Vermiculite.

Le diagramme des RX de la casse rouge est illustré par la figure 3. L'analyse spectrale indique qu'elle est composée de Quartz( $\mathrm{SiO} 2)$, CalciteCa(CO3), Kaolinite (Al2Si2O5(OH)4), Illite [(K,H3O)Al2Si3 AlO10 $(\mathrm{OH}) 2]$ et vermiculite $[(\mathrm{Mg}, \mathrm{Al}) 3(\mathrm{Si}, \mathrm{Al}) 4 \mathrm{O} 10(\mathrm{OH}) 2,4 \mathrm{H} 2 \mathrm{O}]$.

Il révèle principalement la présence de trois pics intenses, le premier correspond à la Quartz et Vermiculite, le deuxième à un mélange de Quartz, Illite, Kaolinite et Vermiculite, le troisième correspond au Calcite et Kaolinite ce qui implique que la casse rouge est hétérogène. Ces résultats sont en parfait accord avec les observations au microscope. La fraction argileuse de notre matériau est constituée de Quartz et de Calcite comme impureté majeure dans notre échantillon. 


\subsection{Caractérisation physique du nouveau matériau composite CR}

Pour tous nos échantillons, le module d' Young est obtenu à partir des courbes de compression avec régression linéaire en exploitant le logiciel Statgraphics.

Nous citons ici l'exemple des échantillons CR à $15 \%$ et à différentes épaisseurs : $6 \mathrm{~mm}, 10 \mathrm{~mm}$ et $15 \mathrm{~mm}$ et les résultats du module d'Young sont regroupés dans le tableau 3.

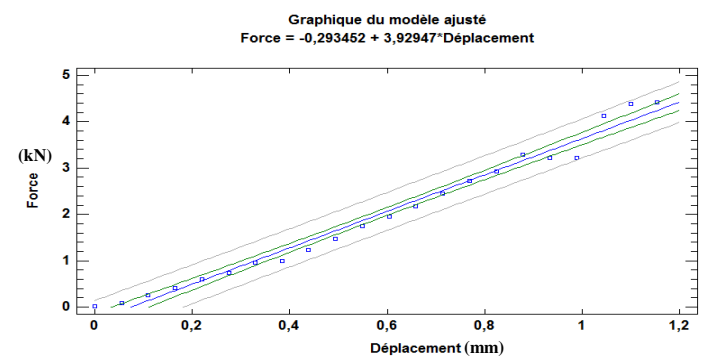

Fig. 4. Graphe du modèle ajusté. Matériau composite CR 15\% (D: 20mm /E: 6mm)

Graphique du modèle ajusté
Force $=-0,129994+4,06943^{*}$ Deplacement

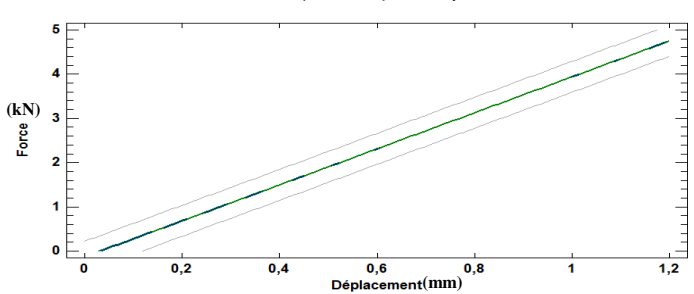

Fig.5. Graphe du modèle ajusté. Matériau composite CR 15\% (D: 20mm /E: 6mm)

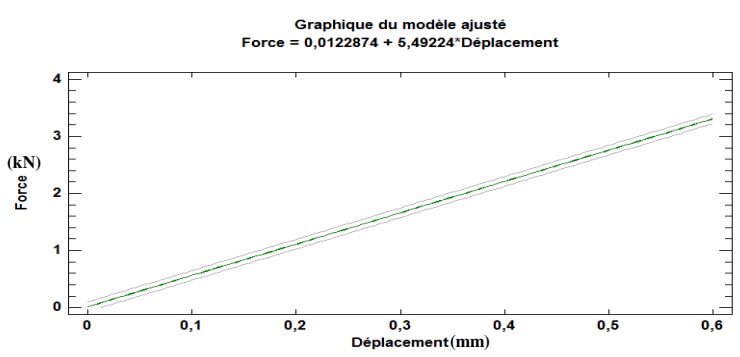

Fig.6. Graphe du modèle ajusté. Matériau composite CR 15\% (D: 20mm /E: 6mm)

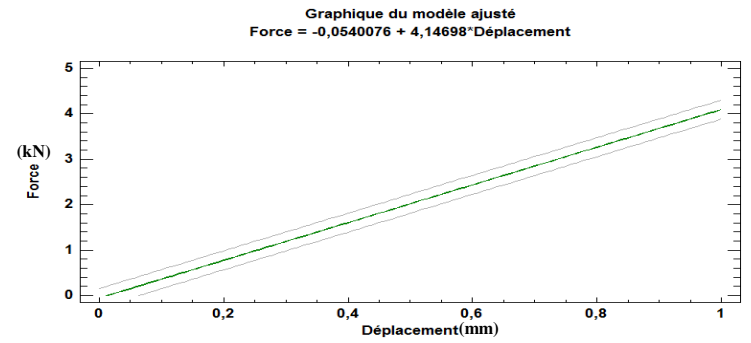

Fig.7. Graphe du modèle ajusté. Matériau composite CR 15\% (D: 20mm /E: 10mm)

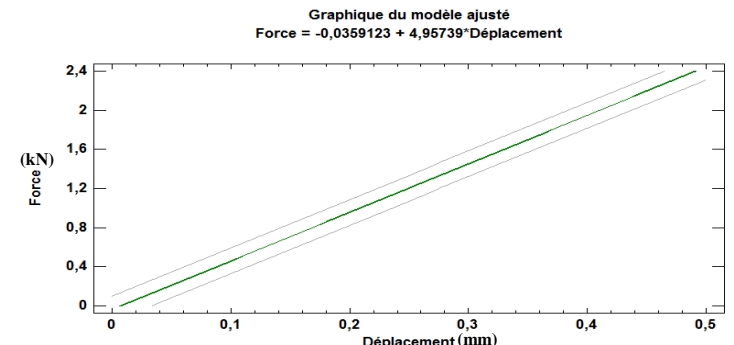

Fig.8. Graphe du modèle ajusté. Matériau composite CR 15\% (D : $20 \mathrm{~mm} / \mathrm{E}: 10 \mathrm{~mm}$ )

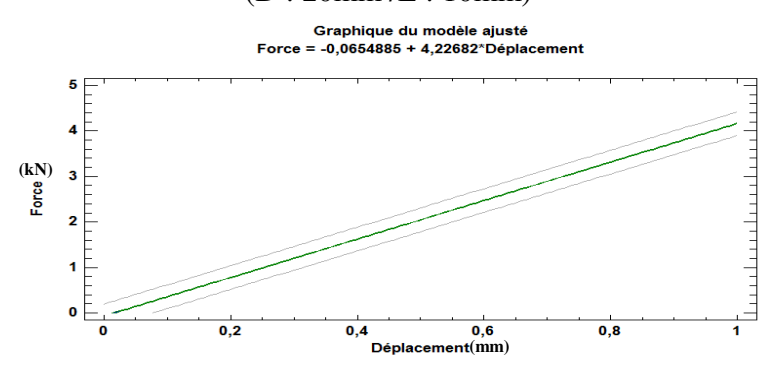

Fig.9. Graphe du modèle ajusté. Matériau composite CR 15\% (D: 20mm /E: 15mm)

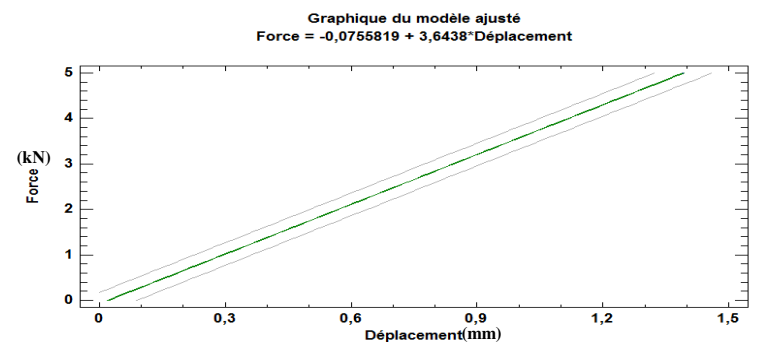

Fig.10. Graphe du modèle ajusté. Matériau composite CR 15\% (D: 20mm /E: 10mm)

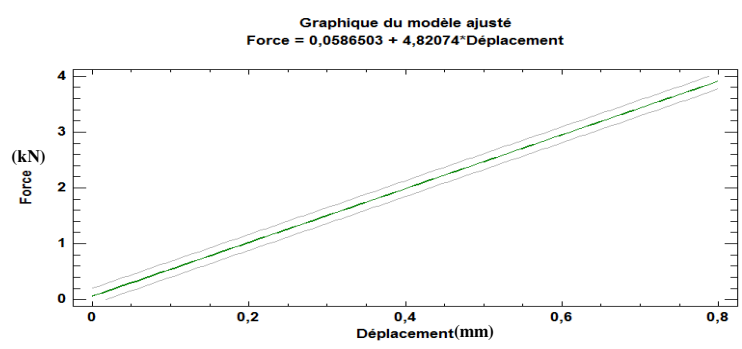

Fig.11. Graphe du modèle ajusté. Matériau composite CR 15\% (D : 20mm /E : 15mm).

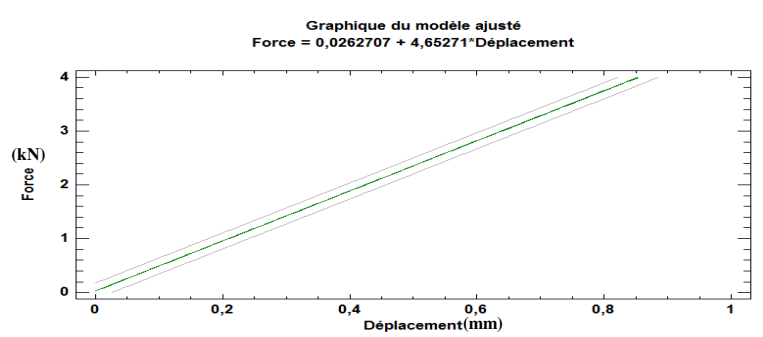

Fig.12. Graphe du modèle ajusté. Matériau composite CR $15 \%$ (D : $20 \mathrm{~mm} / \mathrm{E}: 15 \mathrm{~mm})$ 
Tableau 3. Evaluation du module d'Young pour le matériau composite CR15\%

\begin{tabular}{|l|l|l|l|}
\hline $\begin{array}{l}\text { Matériau } \\
\text { composite } \\
\text { Module d' Young } \\
\text { (E) en Mpa }\end{array}$ & $\begin{array}{l}\text { CR 15\% } \\
6 \mathrm{~mm}\end{array}$ & $\begin{array}{l}\text { CR 15\% } \\
\text { Epaisseur }\end{array}$ & $\begin{array}{l}\text { CR 15\% } \\
\text { Epaisseur } \\
15 \mathrm{~mm}\end{array}$ \\
\hline $1^{\text {er }}$ Essai & 74,90 & 131,84 & 173,88 \\
\hline 2éme Essai & 77,57 & 157,64 & 230,25 \\
\hline 3éme Essai & 104,90 & 134,39 & 222,13 \\
\hline
\end{tabular}

Le même processus de caractérisation mécanique a été suivi pour les autres échantillons CR 30\% et CR 50\%

\section{Synthèse}

L'évolution du module d'Young a été évalué pour les différents composites CR 15\%, CR30\% et CR 50\%.

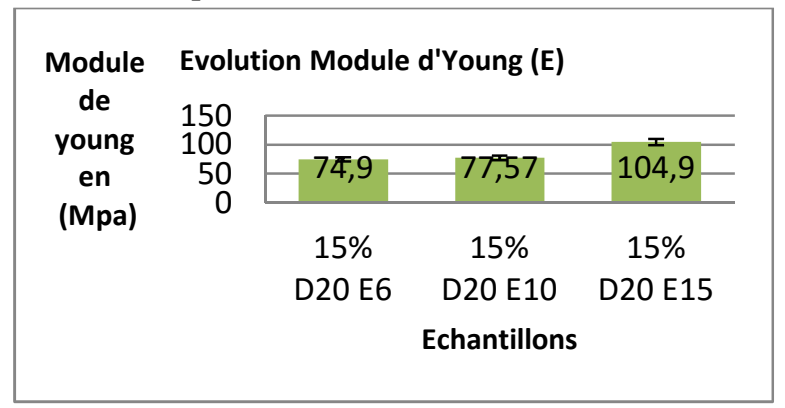

Fig.13. Evolution du module d'Young pour le CR 15\%.

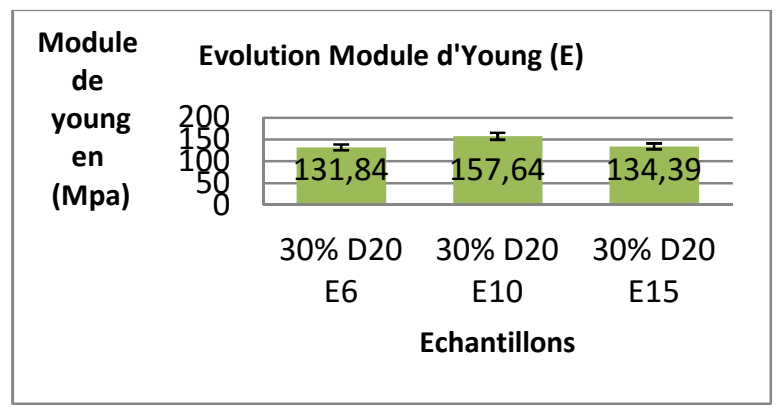

Fig.14. Evolution du module d'Young pour le CR 30\%.

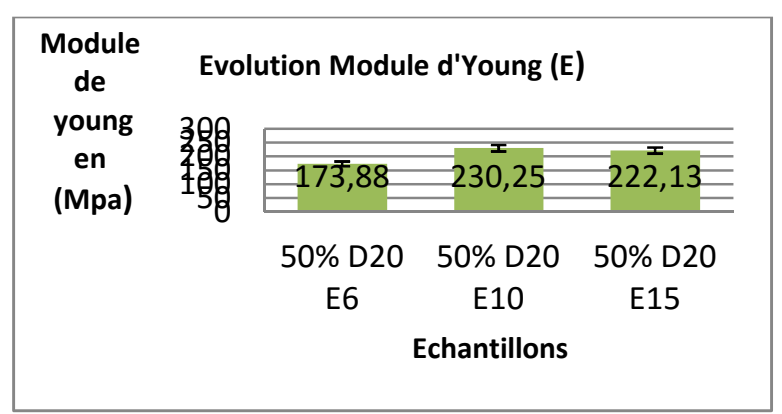

Fig.15. Evolution du module d'Young pour le CR 50\%.
D'après ce graphe, on peut conclure que le renforcement par la casse céramique a un effet direct et positif sur les propriétés mécaniques du nouveau matériau composite. Ceci se traduit par un module d'Young élevé.

\section{Conclusion}

Les techniques expérimentales utilisées nous ont permis de mettre en évidence les propriétés de ce nouveau matériau composite CR.

Ce nouveau matériau composite $\mathrm{CR}$ représente une solution durable et environnementale par la valorisation des déchets BTP.

Les résultats de la présente étude ont mis en évidence la performance et la rigidité du nouveau matériau composite CR traduite par l'évolution du module d' Young. Ce dernier variait progressivement avec la teneur de la charge casse rouge et de l'épaisseur de l'échantillon CR.

\section{References}

1. [1] D.Berthelot, Masson 1996

2. [2] J.-J.Barrau, S.Laroze, Collection Résistance des matériaux et structures, Edition Masson, Paris, 1987.

3. [3] B.Harris, Edition B. Harris, University of Bath, UK, 1983.

4. [4] J. M. Haussounne, Technique de l'ingénieur, E1820, 2 (1996).

5. [5] S. Liebus, Thèse doctorat, Université de Limoges (2003).

6. [6] E. K. Akdogan, A. Safari, J. Appl. Phys, Vol 101, No 6, p 4114, 4121, (2007).

7. [7] N. M. Hagh, B. Jadidian and A. Safari, IEEEUFFC Transaction, vol 55, No 1 , p 214,224 (2008).

8. [8] V. A. Isupov, Soviet. Phys. Solid. State, Vol 12, No 5, p 1084, 1088, (1970).

9. [9] Daniel Michel, Bulletin de L'Union des Physiciens N790 (1997) .

10. [10] K. L.Konan, J.Soro, J.Y.Y.Andji, S.Oyetola, G.Kra, J. Soc. Ouest-Afr. Chim. 30,29. (2010)

11. [11] O.Touret, C. H.Pons, D.Tessier, Y.Tardy, Clay Minerals 25, 223. (1990)

12. [12] H.Zangue Adija, Univ. Lorraine. Eco. Doc. Scien. Et Ing des Ress, Proc, Prod et Env. 132 (2012).

13. [13] A.Aarfane, A.Salhi, M.El Krati, S. Tahiri, M.Monkade, E.K.Lhadi, M.Bensitel, J. Mater. Environ. Sci. 5 (6) 1928(2014).

14. [14] A.Arib, A.Sarhiri, R.Moussa, T.Remmal, C. R. Gomina, Chimie 10,504 (2007).

15. [15] E.Kohler, Univ. Evry Val d'Essonne. Eco. Doc. Phys. Chim, (2005). 